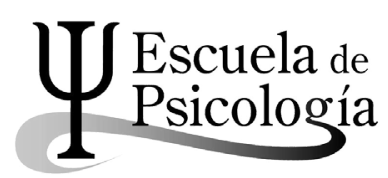

Wímb lu, Rev. electrónica de estudiantes Esc. de psicología, Univ. de Costa Rica. 10 (1): 47-56, 2015 / ISSN: 1659-2107

\title{
MÁS ALLÁ DEL SÍNTOMA. ALGUNOS APUNTES SOBRE EL TRATAMIENTO DEL TRASTORNO DE DÉFICIT ATENCIONAL CON HIPERACTIVIDAD
}

Beyond Symptom. Some notes about the treatment of the Atention Deficit and Hiperactivity Disorder

Daniel Fernández Vásquez*

Resumen: La concepción y el tratamiento habituales del Trastorno de déficit atencional con hiperactividad, principalmente guiados por el DSM-IV, han llegado a causar distintos fenómenos clínicos y sociales en el diagnóstico de este. La perspectiva psicoanalítica del síntoma y la línea que lleva este tipo de clínica pueden brindar una opción que logra curar al paciente cuyos síntomas son ubicados dentro de este trastorno, evitando las faltas que puede traer el intento de normalización hacia el que nos guían las terapias basadas en el DSM-IV o en el cambio de conductas.

Palabras clave: Trastorno de déficit atencional con hiperactividad (TDAH), síntoma, tratamiento farmacológico, tratamiento psicoanalítico, normalización.

Abstract: The usual conception and treatment, guided by the DSM-IV, and used for the Atention Deficit and Hiperactivity Disorder, have came to cause different clinical and social situations related to its diagnosis. The psychoanalytic perspective of the symptom and the clinic it guides to, can give us an option for been able to cure the pacient whose symptoms seem to be a part of this disorder. Avoiding the faults that the DSM-IV and behavior change therapies have lead to.

Key Words: Atention Deficit and Hiperactivity Disorder, symptom, pharmacological treatment, psychonalytic treatment, normalization

Estudiante de psicología, Universidad de Costa Rica.

Correo electrónico: dan_kero@hotmail.com.

Recepción: 10/2/2014 Aceptación: 9/2/2015 


\section{Los pecados del tratamiento habitual}

El comportamiento de los seres humanos es completamente variado. Por lo tanto, resulta ilógico pensar que todas las personas deberían adherirse a un comportamiento "normal". Pues la diversidad es una de las principales manifestaciones de toda sociedad. Ha existido un fenómeno dentro de la clínica psiquiátrica y psicológica actual, al que nos referiremos más adelante, y llamaremos sobre-diagnosticación. Este no es más que ese intento, de nuevo ilógico, de algunos por lograr una normalidad. Todo comportamiento que no se aferre a esta será por tanto eliminado. Eliminado y no estudiado ni comprendido. Eliminado y no analizado. Esta conducta (la de normalización), menciona Freud (1937), es sospechosa de pertenecer a la creencia de quienes consideran que las neurosis (o cualquier otro tipo de comportamiento considerado negativo) son algo que no tiene derecho a existir. Aún más, son estas mismas creencias las que buscan que todos lleguen a ese "yo normal, que como la normalidad en general es solo una ficción ideal" (Freud, 1937, p. 237). Esta ficción ideal, suele ser creada quizá por las personas para mantener el orden social. O quizá otros tantos órdenes que no serán profundizados en este ensayo debido a que por su complejidad, requerirían una dedicación absoluta.

En su búsqueda por eliminar dichos comportamientos, los que no se ajustan a lo socialmente requerido, la medicina ha pecado en repetidas ocasiones. De estas se mostraran aquí, las que a consideración del autor, podrían ser llamadas las principales faltas. La primera, es la sobre-diagnosticación ya mencionada. Esta consiste en agregar dentro de un grupo "patológico" a personas que no pertenecen al mismo o considerar como patológicas, conductas que no son las deseadas. Se desvía la atención de diversas situaciones en la vida de las personas cuya raíz probablemente estaba en otro lugar, o que quizá ni siquiera eran razón de tratamiento. Simplemente eran diferentes. La segunda, la demanda de inmediatez en la eliminación de lo que se considera patológico, lo cual nos lleva a un tratamiento farmacológico rápido y como veremos más adelante, no tan efectivo e incluso algunas o muchas veces peligroso. La necesidad de los fármacos para eliminar síntomas rápidamente, ha provocado toda una generación de seres humanos farmacodependientes, que en muchísimos casos podrían haber prescindido de estos. La tercera, y la que podría ser quizá la más grave, es la despersonalización de lo que se hace. Esta implica la desvinculación de la persona con la forma en que se comporta y lo que con el entendimiento de sí misma podría ser capaz de hacer. Esto provoca un desconocimiento propio de la persona, de sus males y su más inmediata realidad, pasada y presente. Ya hace casi un siglo, Freud se refirió a esta despersonalización, en su momento por parte de los médicos y que actualmente se ve repetida también por muchos psicólogos. "Conocen muy poco a los neuróticos, los oyen con tan poca atención que no han 
enajenado la posibilidad de extraer algo valioso de sus comunicaciones y hacer de estas, observaciones en profundidad" (1917, p.224). La facilidad y la comodidad que brindan los tratamientos rápidos e impersonales han provocado que la mayoría no los cuestione, y se adecúen a ellos, los tomen como lo que se debe hacer y como la forma de ser "normales". Se remite la génesis de los distintos problemas, a la genética y la biología de la persona, con esto se brinda una explicación sencilla y que no compromete la comodidad del diagnosticado. Estos tratamientos que hacen uso de esta metodología nos remiten a la herencia, y así nos brindan una etiología general y remota, en lugar de buscar una causación más próxima (Freud, 1917).

Finalmente podemos decir que lo que llaman el Trastorno de Déficit Atencional e Hiperactividad (TDAH) (APA, 1995) consiste en una serie de síntomas que algunas veces generan un descontento en la persona que los manifiesta, y otras más, en las que la rodean. Especialmente si el consultante que presenta los síntomas es un menor o una menor en ambiente escolar. Cuando estás conductas verdaderamente significan un problema para la persona, cuando no logra controlarlas, es cuando se opta por buscar ayuda de un tercero (aunque muchas veces el "problema" aqueja a alguien que no padece los síntomas, por ejemplo maestros y maestras). En gran cantidad de casos, el tercero (en este caso el médico o la médica) utiliza la medicina y receta fármacos que en teoría curarían los síntomas. Menciona Le Gaufey (s.f) que en casos como este, en que se llegara a la "cura", "podría ser que acontezca, no exactamente la salud, sino el fin de un síntoma, lo que no es nada" (p. 2).

\section{Algunos datos e historia del TDAH y otros diagnósticos}

El trastorno de déficit atencional con hiperactividad, mejor conocido en la sociedad costarricense como "DA" (probablemente por nuestra tendencia a recortar los nombres de las cosas), es ya un viejo conocido de todas y todos los profesionales que se encuentran relacionados al área de la educación o la salud mental. Según datos del Manual Diagnóstico y Estadístico de los Trastornos Mentales IV (DSM-IV por sus siglas en inglés), el cual es uno de los manuales más utilizados a nivel mundial en el área de la psiquiatría y recientemente se encuentra liberando su quinta versión, el TDAH consiste en "un patrón persistente de desatención y/o hiperactividad-impulsividad" (APA, 1995, p. 101). Si nos pusiéramos a contar el listado de síntomas presentes en dicho manual, se notaría que hay en total dieciocho, los cuales van desde "a menudo no presta atención suficiente a los detalles", hasta, "a menudo "está en marcha" o suele actuar como si tuviera un motor" (APA, p. 106-107). La relatividad para la que se pueden prestar estos síntomas ha llevado a que se den distintas situaciones un tanto 
interesantes con el diagnóstico y medicación de este trastorno. Principalmente desde los años noventa hasta la actualidad, época que podemos considerar como el auge de esta enfermedad. Algunas de estas situaciones son mencionadas por Nichols (2009). Por ejemplo, entre 1988 y 1995, las niñas y los niños que eran recetados con fármacos estimulantes (Ritalina) para mejorar su comportamiento en el salón de clase, pasó de medio millón a dos millones y medio. También, los miembros de la sociedad niños y adultos con TDAH (CHADD por sus siglas en inglés), pasaron de ochocientos miembros a treinta y cinco mil en solo esos siete años. Entre los años 2000 y 2003, el consumo de drogas utilizadas para controlar este trastorno, en niños y niñas entre cinco y nueve años, se incrementó en un ochenta y cinco por ciento, y ya para el año 2009, más de cuatro millones y medio de niños y niñas se encontraban diagnosticados con TDAH, de los cuales, más de la mitad, se encontraban medicados con estas drogas. En Alemania, este disparo en la venta de la Ritalina, significó un aumento del doscientos setenta por ciento, tan solo entre los años 1997 y 2000 (Leuzinger-Bohleber, Laezer, PfenningMeerkoetter, Fischmann, Angelika y Green, 2011). El DSM-IV menciona que la prevalencia de este trastorno, es decir la cantidad de niñas y niños que lo padecen en el mundo, se encuentra entre el tres y el cinco por ciento y que los datos en adolescentes y adultos son imprecisos (APA, 1995). Aunque según Salomonsson (2011), estudios más recientes dicen que esta cifra ya supera el cinco por ciento.

Este fenómeno presente durante los últimos casi veinte años, del aumento en el diagnóstico del TDAH, no es único de este trastorno y mucho menos extraño. Nichols (2009) menciona lo que él llama "el extraño rol de la moda en los diagnósticos". En este texto, el autor nos muestra datos relevantes en la cuestión de los diagnósticos médicos, principalmente de enfermedades de carácter psicológico. Inicia con un recorrido sobre una enfermedad descubierta en 1869 por un joven neurólogo en Nueva York, Ilamada neurastenia (griego para nervios cansados). En palabras de su descubridor esta enfermedad se trataba de un deseo de fuerza nerviosa. Para el año 1880, la lista de síntomas de este trastorno era suficiente para llenar dos páginas, e incluía aspectos como, pupilas dilatadas, sonidos en los oídos, y manos y pies sudorosos (Nichols, 2009). Distintas opiniones surgieron respecto a la neurastenia, las cuales fueron cambiando para que ya en 1920, otros diagnósticos se hubieran separado de este. Finalmente llegó a ser reconocido como un fiasco médico y científico. Otros ejemplos de esta moda (esto no afirma que no sean síntomas reales en algunas personas) son, el "borderline" en los años 60's y 70's, la anorexia a finales del siglo XIX, y el trastorno de personalidad múltiple entre los años 1970 y 1990, temporada durante la cual las publicaciones sobre este último aumentaron en un seis mil por ciento (6000\%) y se llegó a afirmar que tenía una prevalencia del uno por ciento, igual que la esquizofrenia (Nichols, 2009). 
Volviendo al TDAH en la actualidad, mucha investigación se ha generado alrededor de este trastorno. Estas han logrado generar diversas hipótesis sobre la base biológica del mismo. A pesar de esto, no hay aún un consenso acerca de las mismas, inclusive ningún estudio funcional o estructural de neuroimagen ha logrado identificar consistentemente la etiología del TDAH (Timimi y Taylor, 2004). La multiplicidad de teorías, junto a la ya mencionada relatividad en los síntomas, genera el constante sobre-diagnóstico ya citado. Con esto y a pesar de la falta de una teoría clara que explique la génesis del trastorno, se ha logrado establecer una droga como la receta general para "curar" a las personas que lo sufren. ¿Por qué curar entre comillas? Diversos estudios han mostrado los efectos nocivos que pueden tener estas drogas a largo plazo (Norman, 2011). Y es este también, un buen punto para cuestionar si cubrir los síntomas con una droga es verdaderamente una cura.

Entonces, si hay investigación que muestra el daño que puede llegar a causar el consumo de fármacos de este tipo, debería ser ya hora de desviar el foco de atención hacia otro tipo de investigaciones aún más recientes. En las cuales, se ha logrado demostrar la eficacia de las terapias no farmacológicas en el tratamiento del TDAH, tanto terapias cognitivo conductuales (Serrano-Troncoso, Guidi y AldaDíez, 2013), así como terapias psicoanalíticas de prevención (Leuzinger-Bohleber et al, 2011) y de tratamiento (Salomonsson, 2011; Norman, 2011). En las terapias cognitivo conductuales, se logra eliminar el fármaco, deshacerse de los efectos a largo plazo. También llegar a "curar" a la persona con TDAH por medio de programas psicológicos y psicoeducativos (Serrano-Troncoso et al., 2013). Pero se debe llegar aquí de nuevo a la cuestión, ¿cambiar un comportamiento puede ser considerado una cura? ¿Será el comportamiento lo que se debe tratar? ¿No sería mejor buscar qué es lo que lo está causando? ¿Y si es cuestión realmente del individuo o más bien del sistema que le rodea? Algunos autores han mostrado un camino diferente, no tratar el TDAH sino a la persona, buscar el por qué. A continuación se realizará una breve exposición de la que se podría llamar una mirada desde el psicoanálisis al tratamiento TDAH, o más bien, al tratamiento psicoanalítico en general que como se verá no habría diferencia.

\section{Una mirada psicoanalítica}

Cada vez que se presenta ante el analista o la analista una persona adjudicándose tener TDAH, o como es en el caso de las niñas y los niños siendo adjudicados por alguien más, la primera persona no puede tomar esta información como un hecho, ya que como veremos en un momento estaría condenando el análisis al fracaso desde antes de empezarlo. Lo más probable es, como ya se mencionó antes, que ya sea quien se adjudica o el encargado o la encargada del 
adjudicado o la adjudicada intervengan demandando una cura a la situación que le aqueja. Ante la demanda de cura, la única opción del analista o la analista es decir "si" aunque no esté de acuerdo. Con este "sí" como dice Le Gaufey, (2000) está casi mintiendo, ya que a lo que realmente se refiere es a un método. Acepta trabajar con las reglas del psicoanálisis y no de la manera que el demandante pide.

Por lo tanto, se inserta el método psicoanalítico para buscar la cura, recordando que no se trata de escuchar e interpretar solamente (aunque siguen existiendo muchos que creen que es así). Al iniciar, y a lo largo de todo lo que dure el tratamiento el analista o la analista debe cuidarse de varias situaciones, que podrían llevar su atención a la eliminación del síntoma, y no al análisis. Al llegar una persona al analista o la analista, la cual presentara síntomas de lo que ha sido llamado TDAH (lo que sea que esto signifique), el primero o la primera deben saber que su manera de abordar el problema que se le presenta, no puede diferir del que existiera ante alguien que llegara porque manifiesta un deseo insaciable de comer (por mencionar algún ejemplo). ¿Por qué? Porque si llegara el analista a cometer el error de dejarse guiar por un síntoma en especial, y por intentar curarlo directamente, provocaría de nuevo el error que ya cometen los otros tipos de terapia (y algunos o muchos psicoanalistas), el cual sería quizá llegar a anular el síntoma. Y como ya dijimos anteriormente, citando a Le Gaufey (s.f.), esto no sería nada. Casi un siglo antes de Le Gaufey, ya Freud también nos prevenía sobre esto y de cómo si el analista se dejaba llevar por el empleo científico y las necesidades de este, estaría poniendo el peligro el éxito del tratamiento, porque a partir del momento en el que el analista tensa adrede su atención, corre el riesgo de no encontrar más de lo que ya sabe (Freud, 1912).

Aparte del caso de la demanda, también podría el analista o la analista encontrarse cautivada o dirigida por las distintas publicaciones existentes con respecto a la etiología de diversos trastornos. Ante esta situación se puede seguir el consejo que Zabarenko, (2011) da con respecto a cómo el analista o la analista debe posicionarse ante estas hipótesis:

Es importante mantener en mente las correlaciones biológicas de los distintos trastornos, pero el analista no puede tomar una posición sobre una etiología de la forma que esta es definida en las ciencias naturales. Este solo puede preocuparse por los síntomas, de la manera que lo haría con cualquier síntoma en la consulta Estos expresan conflictos internos, y la forma de la persona de externalizarlos. No importa que rama teórica utilice el analista, este debe mantener un marco analítico y una actitud óptima (p.6).

Solo manteniendo esta actitud óptima podría el analista llegar a encontrar la relación entre los comportamientos presentados y los conflictos internos que nos menciona Zabarenko, y que también Salomonsson (2011) y Norman (2011) nos señalan como uno de los principales hallazgos de sus estudios. 
Por su lado Salomonsson (2011), afirma que entre los diversos beneficios que puede tener el tratamiento psicoanalítico en niños y niñas diagnosticadas con TDAH, están el crecimiento emocional del niño o la niña y nuestro avance en el conocimiento sobre el mundo interno de estas y estos. Durante sus conversaciones con Nic (caso expuesto en el artículo de referencia), él llega a encontrar una fuerte relación entre el objeto interno y los comportamientos presentados. Y menciona en sus conclusiones, que sería posible quizá, ignorar estas relaciones y mantener la posición de que esto se da por una base neurológica o que es un simple reflejo de las dificultades cognitivas, pero aún así se debe enfatizar el hecho de que estos comportamientos son provocados por factores internos y que el psicoanálisis puede ayudar a descubrir cuáles son. Además, señala, que las capacidades cognitivas y lingüísticas del niño, son a veces hasta deslumbrantes, lo cual le fue posible observar varias veces durante las conversaciones que mantuvieron en sus sesiones. Solo por mencionar un ejemplo, se da un caso en que Nic (un niño de diez años y medio) habla sobre los campos de aplicación innecesarios de la bola de un lapicero (Salomonsson, 2011). Este analista nos muestra como el análisis puede de verdad encontrar la causa de los comportamientos que aquejan al niño. Vale mencionar que luego de casi tres años de análisis, Nic ya había sido transferido de su aula particular a un grupo con todos los demás niños y niñas, aunque aún mostrara algunas veces problemas de hiperactividad y desatención.

Muy similares fueron los resultados y hallazgos que obtuvo Norman (2011) cuando planteó la pregunta ¿es el TDAH un desorden neurológico real o solo una colección de comportamientos sintomáticos psicosociales? Al investigar esta cuestión el autor plantea una interesante cuestión, y esta es: "acaso si se tratara de un componente neurológico entonces el niño o la niña debería ser incapaz de concentrarse en una tarea, cualquiera que esta fuera por un largo intervalo de tiempo". Situación que claramente no sucedía. Por lo tanto descarta la hipótesis neurológica como la génesis. Al exponer el caso de Randall, un niño llevado a análisis por sus padres quienes no querían que fuera sometido al consumo de drogas, el analista llega a la misma conclusión que Zabarenko (2011) y Salomonsson (2011). Encuentra que no solo en el caso de Randall sino en prácticamente todos los casos de niñas y niños etiquetados con TDAH con quienes ha trabajado, existe un factor interno que era exteriorizado como hiperactividad (Norman, 2011). Finalmente el autor afirma, que lo más importante que logra demostrar es que la medicación no fue necesaria para curar al niño, y que no lo ha sido en ninguno de los casos que él ha tratado.

Esta conclusión a la que llega Norman (2011), nos permite abrir un par cuestiones que señalamos al inicio y que sin mencionarlas no me podría dar por satisfecho. Estas son la despersonalización y la intención de encontrar "curas" rápidas a las diversas situaciones que afectan nuestra vida, principalmente las emocionales y de carácter podría decirse "mental". Estas dos cuestiones deben 
ser totalmente evitadas en el análisis, tanto como el enfoque en el síntoma ya que nos llevarían al mismo destino (el fracaso). El camino que se debe seguir en el análisis debe ser guiado por la especificidad de cada caso. Precisamente porque se debe encontrar el sentido verdadero de los síntomas, porque como señala Freud (1900), estos aún si son iguales, en diferentes contextos y personas pueden suponer una génesis distinta. Debido a esto es imposible brindar una receta que permita enseñarnos la manera de actuar cuando se presentara " $x$ " 0 "y" síntoma. Entramos aquí a lo que Freud llama "una extraordinaria diversidad de las constelaciones psíquicas intervinientes, la plasticidad de todos los procesos anímicos y la riqueza de los factores determinantes" (Freud, 1913, p.125). Todo esto integrado con la divergencia existente entre las génesis de los diversos síntomas se opone completamente a una mecanización de la técnica. Así mismo al finalizar su caso con Randall, Norman (2011) nos señala la imposibilidad de que una sola talla pueda quedar a todos, los tratamientos basados en un manual y las medicaciones no pueden, de manera efectiva o segura hacer más que suprimir el síntoma, en el mejor de los casos. Y como ya mencionamos una y otra vez, esto no sería nada.

\section{A modo de cierre}

Al hablar de síntomas de desatención e hiperactividad, quisiera mencionar que existe una preferencia del autor a llamarlos así y no TDAH. Esto, entre muchas razones, por las consecuencias que puede tener una etiqueta, tanto para la persona etiquetada como para las que le rodean. Otra razón, igual de importante, es lo que ya ha mencionado Norman (2011) sobre el término TDAH. Él lo llama un término "bolsa" que permite cubrir cualquier comportamiento que causa inconveniencia a algunos adultos. Ya en algún momento llegué a mostrar que existe una tendencia a la sobre-diagnosticación dentro de la práctica clínica, la cual no hace más que patologizar comportamientos que simplemente, no son los deseados por el sistema, el cual considera como negativa cualquier cosa que sea extraña o que no sea controlable. El TDAH no podría ser considerado más que un intento de un sistema, valga mencionar poco funcional, por re-imponerse como correcto. El pretender mantener a un niño sentado en una silla durante horas escuchando lo que un adulto, probablemente aburrido quiere decirle, esto es simplemente ilógico. En algún momento escuché a alguien decir que no existe el TDAH solo maestros y maestras aburridas.

Pero bueno, está bien. Existe la posibilidad de que existan niños y niñas (jamás más de un $5 \%$, me atrevería a poner los números mucho más abajo como en algunos países europeos) que verdaderamente tengan un comportamiento de desatención e hiperactividad que no logran controlar y que les aqueja 
profundamente. Para estos casos, el psicoanálisis, ha demostrado ser eficaz. Con este se logra, no solo ayudar al niño o niña a dejar estos comportamientos, sino que más que eso, logra encontrar de donde provenía y con ello ir más allá del síntoma, sin esconderlo con el uso de fármacos que suelen empeorar muchas veces la situación. Y por último, pero aún más importante, no intenta normalizarlos, no busca esa normalidad ficticia de la que han hablado varios pensadores como Focault y el mismo Freud. Lo cual sería, considero (y suele serlo en otros tantos tratamientos), un grave error.

\section{Referencias}

American Psychiatric Association (APA). (1995). Manual Diagnóstico y Estadístico de los Trastornos Mentales. MASSON S.A. Barcelona.

Freud, S. (1900). El método de la interpretación de los sueños. Análisis de un sueño paradigmático. La interpretación de los sueños Vol IV (pp. 118-141).

Freud, S. (1912). Consejos al médico sobre el tratamiento psicoanalítico. Escritos técnicos. Vol XII (pp. 107-120).

Freud, S. (1913). Sobre la iniciación del tratamiento (Nuevos consejos sobre la técnica del psicoanálisis, I). Escritos Técnicos. Vol XII. (pp. 121-144)

Freud, S. (1917) Conf. 16: Introducción. Conferencias de Introducción al Psicoanálisis, Vol 15 (pp.223-235).

Freud, S. (1937). Análisis terminable e interminable, vol XXIII, captV-capt-VIII (pp.236-254).

Le Gaufey, G. (s.f.). Reportaje Guy Le Gaufey. (J. Assnadri, Entrevistador)

Le Gaufey, G. (20 de Enero de 2000). Al psiconalista no se le considera profesional. (M. E. Gilio, Entrevistador) Buenos Aires, Argentina.

Leuzinger-Bohleber, M., Laezer, K., Pfenning-Meerkoetter, N., Fischmann, T., Angelika, W., \& Green, J. (2011). Psychoanalytic Treatment of ADHD Children in the Frame of Two Extraclinical Studies: The Frankfurt Prevention Study and the EVA Study. Journal of Infant, Child, and Adolescent Psychotherapy, 10, 32-50. doi:10.1080/15289168.2011.575703

Nichols, D. (2009). Neurasthenia as Allegory: Reflections on the Role of Fashion in Psychiatric Diagnosis. UNIBE: II Jornadas Costarricenses de Psicología Forense. 
Norman, B. (2011). Is ADHD a Real Neurological Disorder or Collection of Psychosocial Symptomatic Behaviors? Implications for Treatment in the Case of Randall E. Journal of Infant, Child, and Adolescent Psychotherapy, 10, 116129. doi:10.1080/15289168.2011.575714.

Salomonsson, B. (2011). Psychoanalytic Conceptualizations of the Internal Object in an ADHD Child. Journal of Infant, Child, and Adolescent Psychotherapy, 10, 87-102. doi: 10.1080/15289168.2011.575711

Serrano-Troncoso, E., Guidi, M., \& Alda-Díez, J. (2013). Is psychological treatment efficacious for attention deficit hyperactivity disorder (ADHD)? Review of nonpharmacological treatments in children and adolescents with ADHD. Actas Esp Psiquiatr, 41(1), 44-51.

Timimi, S., \& Taylor, E. (2004). ADHD is best understood as a cultural construct. British Journal of Psychiatry, 184, 8-9.

Zabarenko, L. (2011). ADHD via Psychoanalysis, Neuroscience, and Cognitive Psychology: Why Haven't We Fielded a Team? Journal of Infant, Child, and Adolescent Psychotherapy, 10, 5-12. doi: 10.1080/15289168.2011.575697

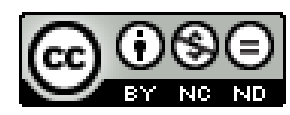

Este obra está bajo una licencia de Creative Commons ReconocimientoNoComercial-SinObraDerivada 4.0 Internacional. 